\title{
VIVÊNCIAS EM UM HOSPITAL PEDIÁTRICO NA CONSTRUÇÃO DE CONHECIMENTO PARA PROFISSIONAIS DE SAÚDE E COMUNIDADE: UM RELATO DE EXPERIÊNCIA
}

\author{
EXPERIENCES IN A CHILDREN'S \\ HOSPITAL IN THE CONSTRUCTION \\ OF KNOWLEDGE TO HEALTH \\ PROFESSIONALS AND COMMUNITY: \\ AN EXPERIENCE REPORT
}

\author{
Tamila do Carmo Araújo* \\ Manoelito Coelho dos Santos Junior**
}

\section{RESUMO}

Este trabalho trata-se de um relato de experiência com ações educativas realizadas em um hospital pediátrico por uma aluna de graduação do curso de Enfermagem da Universidade Estadual de Feira de Santana, que fez parte do programa de extensão "Identificação e análise de interações medicamentosas em prescrições médicas em hospital especializado de Feira de Santana, Bahia". O objetivo foi demonstrar a experiência da estudante em ações educativas voltadas para profissionais de saúde e comunidade. Para os profissionais, foram realizadas palestras com temas relacionados ao ciclo de trabalho. As salas de espera realizadas para a comunidade contaram com a participação de cerca de 20 pessoas por apresentação. Concluiu-se que os profissionais de saúde precisam ser estimulados a buscar cada vez mais, demonstrando que eles desempenham um papel de grande relevância para a instituição e comunidade, e que a comunidade ainda é carente de muitas informações significantes para a saúde.

Palavras-chave: educação; educação continuada; profissional de saúde; comunidade.

\section{ABSTRACT}

This paper is an experience report about the educational actions developed in a children's hospital by an undergraduate student from the Nursing course at the State University of Feira de Santana. The actions were part of the outreach program called Identification and analysis of drug interactions in medical prescriptions in a specialized hospital in Feira de Santana. The aim of this paper was to demonstrate the student's experience in educational actions for health professionals and the community. Lectures about themes related to work cycle were offered to health professionals whereas as waiting rooms were offered to the community with the participation of around 20 people in each presentation. It was concluded that health professionals need to be stimulated more and more, emphasizing that they play a very relevant

*Aluna de Graduação da Universidade Estadual de Feira de Santana (UEFS), BA - Brasil. E-mail: tamila_fsa@hotmail.com 
role for the institution and the community. The study also demonstrated that the community still lack meaningful information about health.

Keywords: education; continuing education; health professional; community.

\section{Introduçáo}

Os trabalhadores de saúde são profissionais indispensáveis para alcançar os objetivos dos serviços de saúde e dos processos de trabalho. Estes necessitam buscar constantemente conhecimentos sobre a prática, atualização técnico- científica e o diálogo com usuários e os demais trabalhadores que integram o serviço (PEDUZZI et al., 2009).

Nos serviços de saúde, os recursos humanos são ainda significativamente marcados por procedimentos de administração de pessoal. As ações educativas são pontuais, centradas em capacitações técnico-científicas fragmentadas, sendo em grande parte desvinculadas das necessidades de saúde (PEDROSO, 2005).

A Educação Continuada (EC) pode ser conceituada como qualquer ocasião que possa oferecer informação, troca de saberes e discussóes que favoreçam o aprimoramento profissional. Foi colocada como avanço e aprofundamento nas formaçóes profissionais (GATTI, 2008), sendo uma maneira de proporcionar o aperfeiçoamento e desenvolvimento dos recursos humanos das instituições. As atividades desenvolvidas pela EC podem aproximar a lacuna existente entre a formação e a real necessidade do sistema, sendo, assim, importantes para consolidaçáo do Sistema Único de Saúde (SUS) (PEDROSO, 2005).

O aprendizado é um processo contínuo, ativo e depende de motivação para ser alcançado. A qualidade dos resultados obtidos após uma EC é de responsabilidade dos organizadores, mas também dos participantes envolvidos nesse processo (MONTEIRO; CHILLIDA; BARGAS, 2004).

A convivência dos profissionais de saúde com a comunidade favorece a deteç̧ão da realidade da clientela. Lidar com usuários dos serviços de saúde envolve práticas, saberes, mitos, tabus e representaçóes que fazem parte da subjetividade coletiva (TEIXEIRA, 2001). Além disso, os profissionais são responsáveis pela transmissão de informaçóes que visem à promoção da saúde.

Nas instituiçôes hospitalares, observa-se que as ações relacionadas à saúde estão voltadas para a promoção de saúde da população e o tratamento das patologias, mas também deve ser destacado o comprometimento destas com a educação de seus profissionais (NETO, 2001).

As instituições de saúde estão em constantes transformaçóes nos seus sistemas e processos, buscando atender as necessidades da clientela, principalmente pelo avanço da tecnologia e ciência (LAZZARI; SCHMIDT; JUNG, 2012). Através da educação continuada, se visam modificações e melhorias nos processos de trabalho para alcançar níveis satisfatórios de qualidade (BEZERRA, 2003), mas muitas instituições encontram dificuldades para executar açôes educativas com seus trabalhadores devido à disponibilidade destes, carga horária, entre outros. Além do que, outra dificuldade é que, no ambiente hospitalar, o trabalho é complexo e exige que os profissionais sejam atualizados e capacitados constantemente (SENHORAS, 2007).

Em face a essas consideraçóes, o presente relato de experiência tem como objetivo demonstrar a experiência de uma estudante de enfermagem de uma Universidade Estadual, entre o $9^{\circ}$ e $10^{\circ}$ semestre, em açôes educativas voltadas para profissionais de saúde e comunidade de um hospital estadual pediátrico público. Este relato de experiência foi desenvolvido durante a vivência da acadêmica no plano de trabalho do Programa de Extensão "Identificação e análise de interações medicamentosas em prescrições médicas 
em hospital especializado de Feira de Santana, Bahia, vinculado à Universidade Estadual de Feira de Santana (UEFS)".

\section{Métodos}

As atividades aqui descritas referem-se ao período de março de 2015 a março de 2016. O presente trabalho foi avaliado pelo Comitê de Ética em Pesquisa (CEP) da Universidade Estadual de Feira de Santana, tendo suas atividades aprovadas conforme parecer do referido CEP (CAAE:11325812.0.0000.0053).

O programa de extensão em foco visou contribuir com o fortalecimento da Farmácia Clínica no Hospital Estadual da Criança (HEC) de Feira de Santana, Bahia. O objetivo foi integrar o Serviço de Farmácia do HEC junto à equipe multidisciplinar de saúde, trazendo benefícios aos membros desta equipe, aos pacientes internados e aos docentes e discentes do Curso de Farmácia, Enfermagem e Medicina.

A identificação e análise de interaçôes medicamentosas nas prescrições médicas foram úteis para a prescrição racional de medicamentos nesta unidade hospitalar, bem como para a prevençáo e/ou minimização da ocorrência de eventos adversos decorrentes destas interaçôes, além de açóes educativas que visaram à segurança do paciente, através do suporte educacional à equipe de saúde e comunidade.

Inicialmente, a proposta para a realização das palestras científicas e salas de espera foi apresentada para a coordenação da instituiçáo, mostrando-se o cronograma e os objetivos das atividades. Posteriormente, buscou-se, dos profissionais envolvidos no processo de educaçáo continuada, quais temas seriam interessantes para serem abordados nas palestras. Foram realizadas pesquisas em artigos científicos sobre as temáticas sugeridas, com o intuito de levantar informaçóes atualizadas sobre os temas abordados nas palestras.

As açôes educativas foram desenvolvidas através de seminários e salas de espera. $\mathrm{O}$ seminário é uma das técnicas de ensino cujo objetivo é proporcionar interaçáo e diálogo, gerando uma troca de ideias entre quem apresenta e que assiste (RANGEL, 2005), além de construir reflexóes e conhecimentos. A sala de espera funciona como um espaço que potencializa as discussóes, reflexóes e posicionamentos, em que as práticas de saúde são maximizadas, além de ser uma forma de amenizar o desgaste físico e emocional que pode ser causado pela espera de algum atendimento (ROSA; BARTH; GERMANI, 2010). É um meio educativo descontraído, informal, para que a comunidade se permita a expor opiniôes e dúvidas, além da aproximação dos serviços de saúde. Ambos instrumentos foram escolhidos por se mostrarem efetivos e possuírem fácil execução.

Os seminários foram realizados no auditório da instituiçáo, em dias da semana diferentes, e em turnos diferentes, com o objetivo de atingir o maior número de profissionais. Para isso, também se avaliaram as escalas de trabalho dos profissionais, priorizando as datas com mais acessibilidade para eles. A duração das palestras foi estipulada em duas horas, sendo os 30 minutos finais destinados a perguntas dos ouvintes. $\mathrm{O}$ tema abordado foi referente à prescrição, dispensação e administração de medicamentos no ambiente hospitalar, enfatizando-se o cuidado na segurança do paciente.

As salas de espera foram realizadas no ambulatório da instituição, por ser um local estratégico, de fácil acesso para comunidade, além de possuir uma grande concentraçáo de pessoas, tornando o momento mais produtivo. As escolhas dos temas foram baseadas 
em sua relevância social e, com isso, foram eleitos os temas de utilizaçáo correta de medicamentos (devido à carência de informaçóes sobre o manejo de fármacos), amamentaçăo (devido à natureza do Hospital), plantas medicinais (devido à carência de informaçóes sobre as mesmas). Utilizaram-se folders, por ser um material simples, objetivo e que as informaçóes contidas podem facilmente ser disseminadas, os quais foram produzidos pela pesquisadora e seu orientador. Com linguagem simples e clara, os folders continham figuras, para se obter um maior esclarecimento, e foram explicados por tópicos de cada tema, e, simultaneamente, experiências eram ouvidas e dúvidas esclarecidas. Cada sala de espera teve duração de aproximadamente 40 minutos.

\section{Resultados e análise}

Os erros na administração de medicamentos podem gerar graves problemas para a saúde, principalmente em virtude da exagerada carga horária dos profissionais. É de fundamental importância a atençáo a esses erros na administraçáo de medicamentos, principalmente para aqueles que trabalham com crianças, por elas possuírem uma fisiologia em formaçáo, respondendo aos medicamentos de uma maneira diferente dos adultos (PAIVA; MOURA, 2012).

Os erros quanto à administração de medicamentos motivaram a realização da palestra sobre a prescrição, dispensação e administração segura de medicamentos. Além do que, atualizar os profissionais e conscientizá-los da importância desses afazeres é fundamental para o paciente. A palestra também auxiliou de forma positiva para o entendimento sobre a qualidade da assistência e segurança do paciente.

O Programa Nacional de Segurança do Paciente (PNSP) surge através da Portaria n529, no ano de 2013, pelo Ministério da Saúde, com o objetivo de contribuir na qualificação do cuidado em saúde, oferecendo uma assistência segura (BRASIL, 2013). A partir da temática, foi induzido aos profissionais a reflexão sobre seu exercício profissional, além de questionamentos de diversos aspectos: realmente conheço os riscos do meu trabalho? Sei reconhecer os riscos dos procedimentos que realizo? O que posso fazer para controlar os riscos? Eu tenho atitudes preventivas? E se acontecer algum acidente? Qual meu papel educativo em minha equipe? Estes questionamentos aumentam, sem dúvida, a criticidade nas açóes dos profissionais.

Foram realizados oito seminários para capacitação profissional, os quais contaram a participação de cerca de 30 profissionais por apresentação. O campo de trabalho na área da saúde apresenta, historicamente, baixa concentração de trabalho masculino, sendo que o setor da enfermagem geralmente responde por volta de $85 \%$ de trabalho feminino, além da equipe de enfermagem representar, em média, $40 \%$ do total dos trabalhadores de saúde (PASTORE; ROSA; HOEMEM, 2008).

Em relação às profissóes, estiveram presentes enfermeiros, técnicos de enfermagem, farmacêuticos e fisioterapeutas. A capacitação desses profissionais foi um fator positivo, devido à sua responsabilidade em prestar assistência direta e indireta aos pacientes, além de desenvolverem uma série de procedimentos que necessitam de concentração, destreza e conhecimento.

Durante os seminários (Figura 1), a estudante interagiu com os profissionais, solicitando respostas a perguntas que foram sendo realizadas, como: quais são os certos 
para a administração de um medicamento? Quais cuidados devem ser tomados para o controle de infecção hospitalar? Vocês conhecem o termo Segurança do Paciente? Essas respostas demonstravam o conhecimento prévio dos profissionais, assim como incentivava a sua participação. Os profissionais presentes receberiam certificado de participação após cada capacitação e a instituição exigia a participação. Esses fatores foram importantes facilitadores, mas observou-se, também, que a exigência foi um fator que dificultou o processo de aprendizagem. Cerca de cinco profissionais por apresentação năo tiveram participação efetiva, não interagiram e mostraram-se desinteressados.

Aos que participaram efetivamente, a apresentação foi produtiva, com construção de conhecimentos, troca de experiências, atualizando os profissionais de saúde da instituição, além do alerta para a qualificação no atendimento dos pacientes.

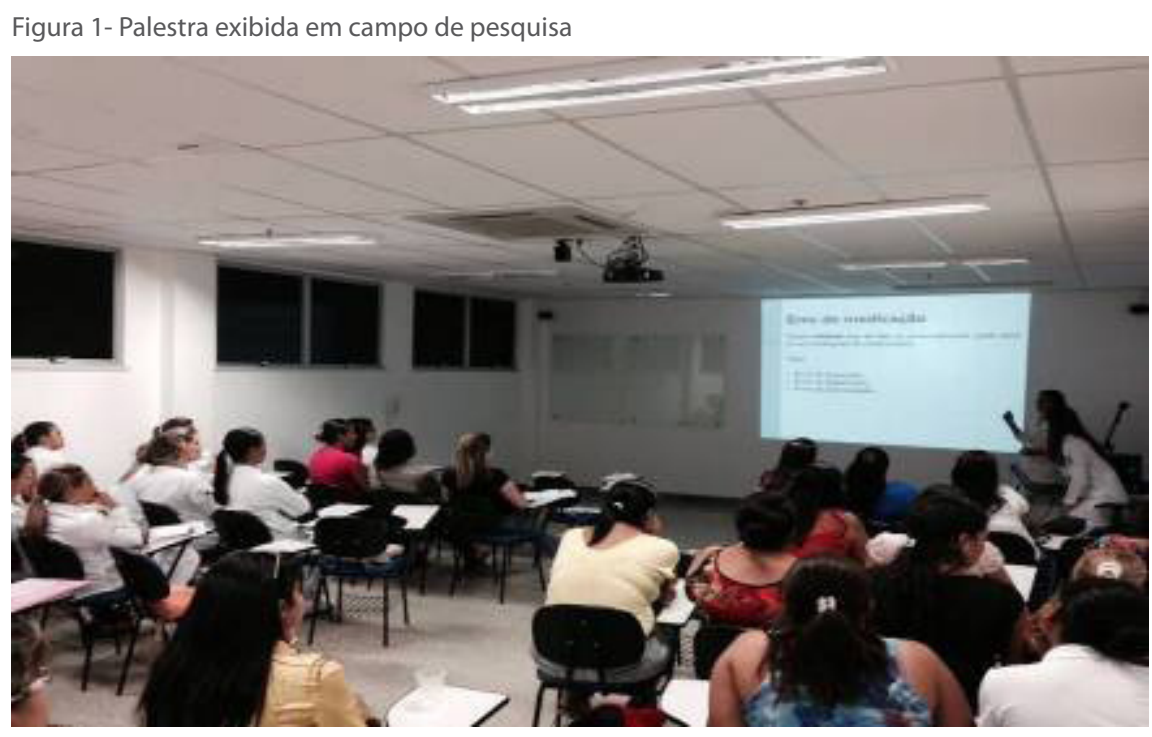

As salas de espera contaram com a participaçáo de cerca de 20 pessoas por apresentação, totalizando 7 apresentações. O primeiro tema abordado foi a automedicação (Figura 2). A automedicaçấo, que já é um hábito na sociedade brasileira, leva cerca de 20 mil mortes no país por ano, a maior parte justificada por intoxicaçóes medicamentosas e reaçóes alérgicas (IVFRJ, 2006). O uso de medicamentos por conta própria pode ser realizado tanto com medicamentos industrializados como caseiros. A prática da automedicação ocorre por diversas razóes, destacando-se a dificuldade para conseguir atendimento médico, a limitação do poder de prescrever, por haver poucos profissionais habilitados, falta de fiscalização adequada, medicamentos subscritos, entre outras (AUTOMEDICAÇÃO, 2001). 
Figura 2 - Folder sobre utilização correta dos medicamentos, entregue em sala de espera
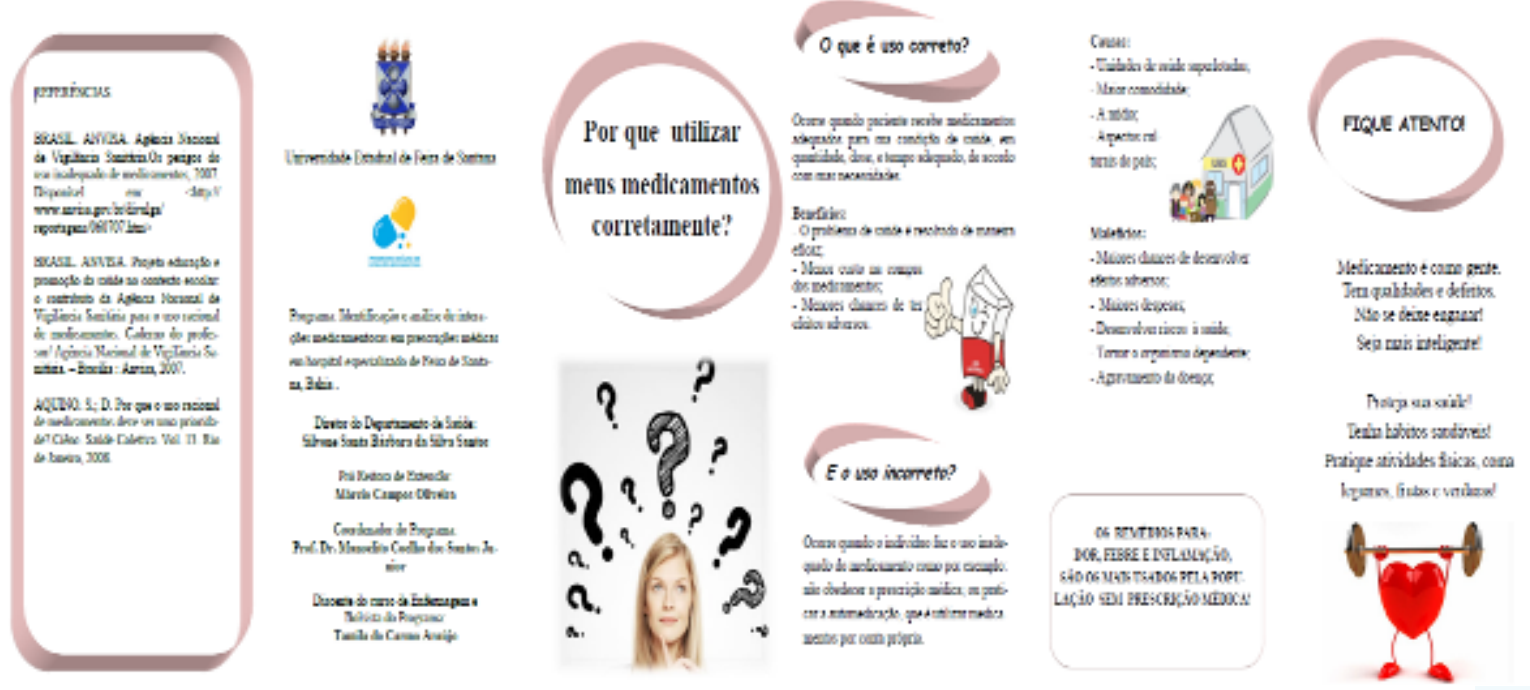

O uso de medicamentos por conta própria foi confirmado pela comunidade, principalmente os analgésicos. Foi possível constatar que alguns pacientes justificaram o uso sem prescrição por ter recebido indicação de outra pessoa, por acreditar que não faria "mal", ou por já ter o medicamento em sua residência. Foi alertado pela estudante quanto aos riscos do uso de medicamentos por conta própria, como por exemplo, o mascaramento de diagnósticos.

Outro tema abordado foi o uso de medicamentos durante a amamentaçáo (Figura 3). Nesse período, se for preciso fazer uso de algum medicamento, é necessário conhecer fatores que determinam a segurança da utilizaçáo; além disso, a composição do leite materno varia de acordo com a fase de lactação, ou até mesmo durante uma mamada. Esses fatores interferem na quantidade de fármacos que são transferidos do plasma para o leite materno, gerando variaçóes nas concentraçóes destes no leite materno (CHAVES et al., 2007).

Figura 3 - Folder sobre medicamentos durante a amamentação, entregue em sala de espera
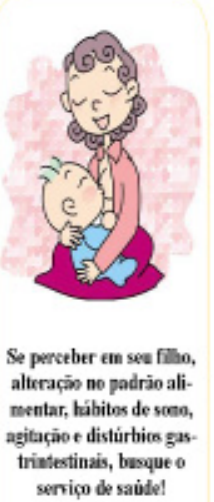
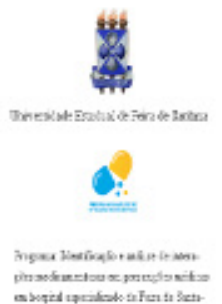

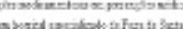
antes.

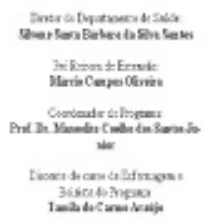

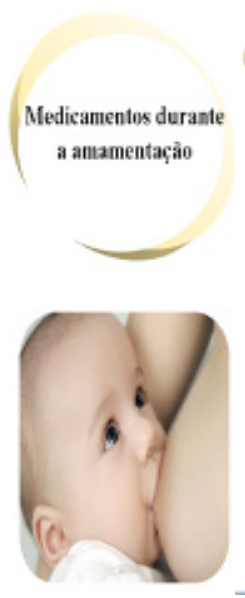

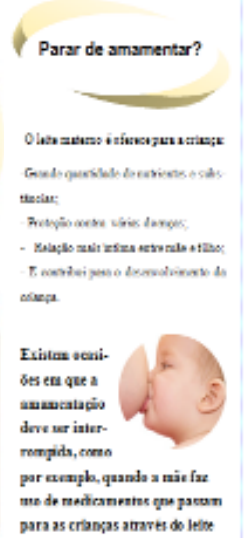

materas:

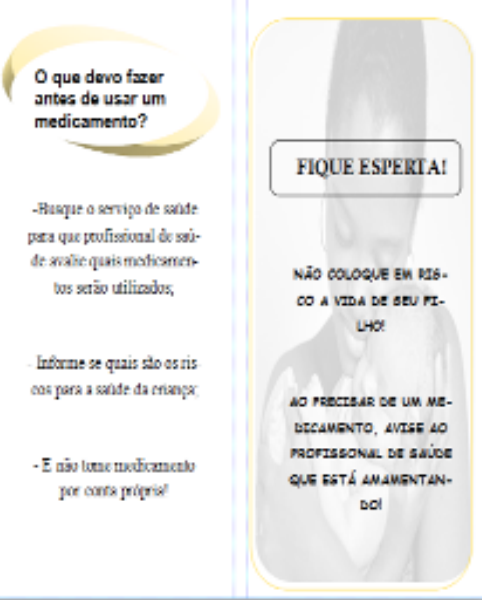


As mulheres presentes nas salas de espera, que ainda estavam no período de amamentação, não sabiam que os medicamentos por elas utilizados poderiam ser passados para a criança através do leite materno, podendo gerar riscos como consequências. Foilhes então orientado a utilizarem medicamentos apenas com prescrição médica, e para as genitoras informarem aos profissionais de saúde que ainda estão ofertando o leite materno para a criança, pois assim os profissionais avaliarão o risco e benefício e prescreverão o que for mais adequado.

Foi abordado também o uso de plantas medicinais (Figura 4). Utilizar as plantas medicinais como forma de tratamento é um costume antigo, as diferentes culturas influenciam na utilização das plantas visando o tratamento de doenças e a manutenção da saúde (PARIZ et al., 2012). O cuidado obtido através de plantas medicinais pode ser favorável à saúde humana desde que quem as utilizam tenha conhecimento sobre a finalidade, riscos e benefícios (BADKE et al.,2013).

Figura 4- Folder sobre plantas medicinais, entregue em sala de espera

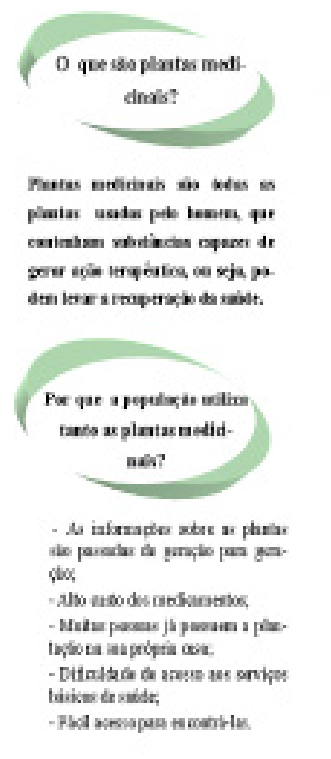

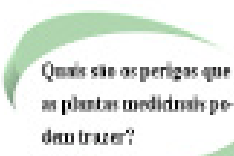

$$
\text { dew truse? }
$$

- Tesiaidak da orzimer

- Asromeno da dosecs

- Gere cove socolonabs paroe aask

- Cuara abuso

Marcene dagenadion

- Interiqir tan dên nxắnusata

que jas foux

- Eationec levar asent
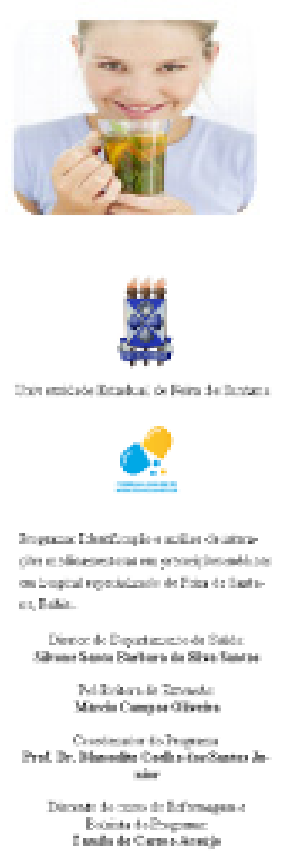

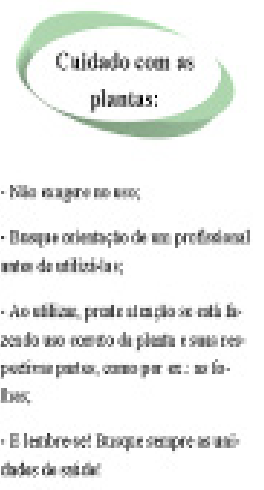

biscocosice
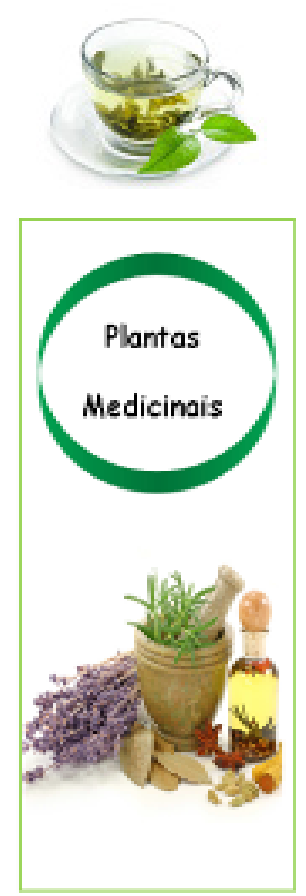

Observou-se que a comunidade utiliza de forma indiscriminada e sem cuidado as plantas medicinais em forma de chá, por ser uma forma "efetiva", barata e fácil de ser 
realizada. Foi explicado que as plantas possuem substâncias, assim como medicamentos, e não devem ser utilizadas sem conhecimento e nem em excesso.

Foi orientado à população, durante as salas de espera, buscar os serviços de saúde, assim como o esclarecimento de dúvidas e hábitos saudáveis, como alimentação rica em frutas e legumes e realizar atividades físicas, além de aproximar e criar vínculo entre o serviço de saúde e comunidade, fortalecendo o SUS.

Um dos fatores que dificultou a concentração da comunidade no momento das salas de espera foi o som causado pelas crianças. Por ser um hospital pediátrico, era difícil se obter um ambiente de silêncio. Outro fator que pode ter dificultado a adesão das informações transmitidas foi a cultura. Cada indivíduo tem seus hábitos e crenças, e isso gera resistência para compreender informaçóes que vão contra os seus pensamentos, principalmente as pessoas idosas.

A maior facilidade veio da própria populaçáo, a qual mostrou-se receptiva, participativa, questionou os aspectos sobre os quais não concordava, contou experiências e esclareceu informaçóes. Importante também destacar que, no fim das apresentações, a satisfaçáo da comunidade foi notada por meio de agradecimentos.

\section{Conclusões}

É de fundamental importância que as instituiçóes hospitalares percebam a necessidade de uma ênfase maior no que se diz respeito à segurança do paciente, oferecendo qualificaçáo para os profissionais, atualizando-os, gerando benefícios não só para a comunidade, mas também para instituiçáo.

Os profissionais de saúde precisam ser estimulados a buscar cada vez mais, seja através de palestras, oficinas, ou qualquer outra metodologia que proporcione apreender de forma prazerosa, demonstrando-se que eles desempenham um papel de grande relevância para a instituição e comunidade.

Conclui-se que a comunidade ainda é carente de muitas informaçôes relevantes para a saúde, mas que estas podem ser transmitidas de maneira fácil, através das salas de espera. Atividades em grupo favorecem o desenvolvimento do indivíduo devido à diversidade e troca de experiências.

Além disso, as salas de espera permitem que a comunidade seja capturada para os serviços de saúde e induzidas a se cuidar mais. Devendo também nesse momento acolher as pessoas e escutá-las, para que percebam seu papel e sua autonomia diante de sua qualidade de vida.

Com o desenvolvimento das atividades, conclui-se também que os estudantes, junto aos orientadores, realizam um papel transformador para o conhecimento, possibilitando uma atuação voltada para os interesses e necessidades da populaçáo, ligados à saúde. Tudo isso proporciona também ao estudante um crescimento pessoal, que interfere diretamente no futuro profissional.

\section{Referências}


BADKE, M.R et al. Plantas medicinais: o saber sustentado na prática do cotidiano popular. Esc. Anna Nery. [Internet], v.15, n. 1, p.132-139, 2011. Disponível em:< http://www.scielo. br/pdf/ean/v15n1/19.pdf> Acesso em: 15/03/2017

BEZERRA, A. L. Q. O Contexto da Educação Continuada em Enfermagem. São Paulo: Lemar e Martinari, 2003.

BRASIL. Ministério da Saúde. Portaria n 529/2013. Programa Nacional de Segurança do Paciente (PNSP). Disponível em:< http://bvsms.saude.gov.br/bvs/saudelegis/gm/2013/ prt0529_01_04_2013.html> Acesso em:21/03/2017.

CHAVES, R.G. et al. Medicamentos e amamentação: atualização e revisão aplicadas à clínica materno-infantil Medicines and breastfeeding: update and revision applied to mother and baby care. Rev. Paul. Pediatr., v. 25, n. 3, p. 276-288, 2007.

GATTI, B.A. Análise das políticas públicas para formação continuada no Brasil, na última década. Revista Brasileira de Educação, v. 13, n. 37, jan./abr. 2008.

IVFRJ. Instituto Virtual de Fármacos do Estado do Rio de Janeiro. Rio de Janeiro, 2006. Disponível em: <http:// www.ivfrj.ccsdecania.ufrj.br/ivfonline/edicao_0012/ automedicacao.html> Acesso em: 15/03/2017.

LAZZARI, D.D.; SCHMIDT, N.; JUNG, W. Educação continuada em unidade de terapia intensiva na percepção de enfermeiras. Rev. Enferm., UFSM, v.2, n.1, p.88-96, 2012.

MONTEIRO, M.I.; CHILLIDA, M.S.P.; BARGAS, E. B. Educação continuada em um serviço terceirizado de limpeza de um hospital universitário. Rev. Latino-am. Enfermagem., v. 12, n.3, p. 541-548, mai./jun. 2004.

NETO, F. J. S. L. Módulo IV - Educação/Trabalho/Profissão. Rio de Janeiro: Fundação Oswaldo Cruz, 2001.

PAIVA, N. A.; MOURA. C. S. Interações medicamentosas Potenciais nas prescrições de Pacientes Pediátricos Hospitalizados. Rev. Bras. Farm., Vitória da Conquista v. 93, n .4, p. 463-468, 2012.

PARIZ, M. A. et al. Uso de plantas medicinais: impactos e perspectivas no cuidado de enfermagem em uma comunidade rural. Rev. Eletr. Enf. [Internet], v.15, n. 4, p. 993, out./ dez. 2013. Disponível em: <https://www.fen.ufg.br/fen_revista/v15/n4/pdf/v15n4a17. pdf $>$. Acesso em: 15/03/2017.

PASTORE, E.; ROSA, L. D.; HOEMEM, I.D. Relações de gênero e poder entre trabalhadores da área da saúde. Florianópolis, 2008.

PEDROSO, V.G. Aspectos conceituais sobre educação continuada e educação permanente em saúde. Mundo Saúde, v.29, n.1, p.88-93, 2005.

PEDUZZI, M. et al. Actividades educativas de trabajadores en la atención primaria: concepciones de educación permanente y de educación continuada en salud presentes en el quehacer cotidiano de Unidades Básicas de Salud en Sao Paulo, Brasil. Interface (Botucatu) [online], v.13, n.30, p.121-134. ISSN 1807-5762, 2009. 
RANGEL, M. Métodos de ensino para a aprendizagem e dinamização das aulas. Coleção: Magistério: Formação e Trabalho Pedagógico. 2. ed. São Paulo: Papirus, 2005.

ROSA, J.; BARTH, P. O.; GERMANI, A. R. M. A sala de espera no agir em saúde: espaço de educação e promoção à saúde. Perspectiva, Erechim. v.35, n.129, p. 121-130, mar.2011.

SENHORAS, E. M. A cultura na organização hospitalar e as políticas culturais de coordenação de comunicação e aprendizagem. RECIIS - R. Eletr. de Com. Inf. Inov. Saúde, Rio de Janeiro, v.1, n.1, p.45-55, jan./jun, 2007. Disponível em:<http://www.reciis.icict.fiocruz.br/index.php/reciis/article/viewFile/45/55>. Acesso em: 25/03/2017.

TEIXEIRA, E.R. Estética e subjetividade no cuidado com o corpo. In: SANTOS, I.; FIGUEIREDO, N. M. A.; DUARTE, M. J. R. A.; SOBRAL,V. R. S.; MARINHO, A. M. (Orgs.). Enfermagem fundamental: realidade, questões, soluções. São Paulo: Atheneu, 2001, p.221-226. 